\title{
Magnetic seeded filtration for the separation of fine polymer particles from dilute suspensions: Microplastics
}

\author{
Frank Rhein, Felix Scholl, Hermann Nirschl \\ Karlsruhe Institute of Technology (KIT), Institute for Mechanical Process Engineering and Mechanics, Strasse am Forum 8, 76131 Karlsruhe, Germany
}

\section{H I G H L I G H T S}

- Efficient removal strategies for microplastics are lacking.

- Magnetic seeded filtration showed high separation efficiencies ( $95 \%$ ) for dilute suspensions.

- Electrostatic interactions between particles were shown to be process determining.

- A parameter study identified relevant process variables and yielded clear application guidelines.

- The measurement of agglomeration kinetics enables further optimization.

Keywords:

Magnetic seeded filtration

Microplastic

Hetero agglomeration

Solid-liquid separation
G R A P H I C A L A B S T R A C T

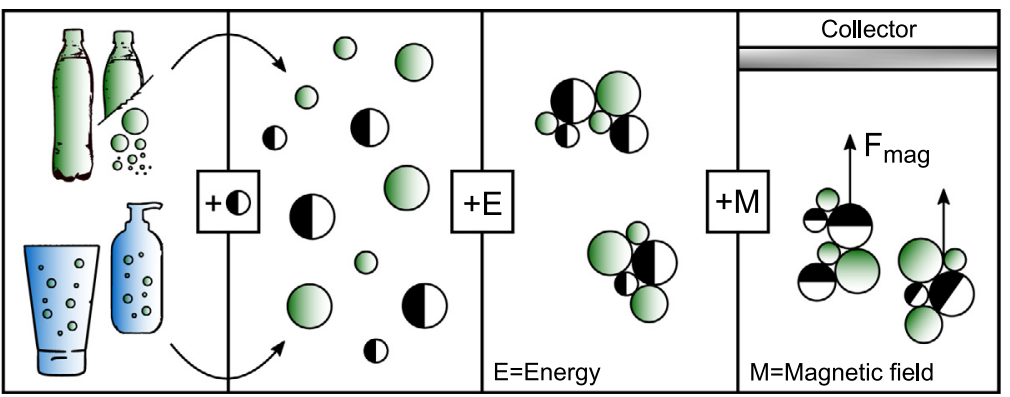

\section{A B S T R A C T}

Microplastics have become a highly discussed topic: Whether through the decomposition of larger plastic waste or direct usage on a small particle scale, microplastics find their way into the natural ecosystem with uncertain consequences: In particular, the ingestion and therefore the introduction of these particles into the food web (often carrying adsorbed chemicals) poses still not fully understood hazards for man kind. This work focuses on the separation of such particles from aquatic suspensions and evaluates the viability of magnetic seeded filtration in this field. In this study it could be shown that it is generally pos sible to separate fine polymeric particles from dilute suspensions in a highly effective manner. A broad range of process conditions were investigated with separation efficiencies reaching up to $95 \%$. The $p H$ and ultimately the surface charge of the particles were identified to be the crucial process relevant parameters. More exactly, the surface potentials should be either small or oppositely charged leading to a diminishing or even attractive electrostatic interaction. However, when both agglomeration partners exhibit an electrostatic repulsion, a thorough parameter optimization nevertheless yielded high separa tion efficiencies. In particular, the ionic strength and magnetic seed concentration proved to be determin ing factors in this case. Lastly, the process kinetics were investigated and a limited exponential growth character was observed which could be modeled with basic equations from agglomeration theory under simplifying assumptions. This knowledge further assists the selection of an optimal parameter set for a process engineering application.

\section{Introduction}

Plastic is a collective term for synthetic or semi synthetic polymer products and is deeply rooted in today's language. LIFE magazine published an article called "Throwaway living" in the year 


\begin{tabular}{|c|c|c|c|}
\hline \multicolumn{4}{|c|}{ Nomenclature } \\
\hline$A_{H}$ & HAMAKER constant $[J]$ & $R^{2}$ & coefficient of determination [ ] \\
\hline$A_{\lambda}$ & absorbance at wavelength $\lambda\left[\mathrm{cm}^{-1}\right]$ & $r_{i}$ & radius particle $i[\mathrm{~m}]$ \\
\hline$a_{\text {wire }}$ & wire diameter $[\mathrm{m}]$ & $T$ & temperature $[\mathrm{K}]$ \\
\hline$B$ & magnetic flux density $[\mathrm{T}]$ & $T_{N M}$ & separation efficiency for the non magnetic particles [\%] \\
\hline$C_{1}$ & agglomeration rate constant $\left[\mathrm{s}^{-1}\right]$ & $t_{\mathrm{A}}$ & agglomeration time [s] \\
\hline$C_{2}$ & agglomeration limit constant [\%] & $V_{p}$ & particle volume $\left[\mathrm{m}^{3}\right]$ \\
\hline$c_{v, M}$ & volume concentration magnetic particles [vol\%] & $X_{i}$ & normalized experimental factor [ ] \\
\hline$c_{v, N M}$ & volume concentration non magnetic particles [vol\%] & $x_{50}$ & mean diameter $[\mathrm{m}]$ \\
\hline & absolute effect of factor $X_{i}$ on the separation efficiency & $\alpha_{i, j}$ & collision efficiency between $i$ and $j\left[\mathrm{~m}^{-3}\right]$ \\
\hline & {$[\%]$} & $\beta_{i, j}$ & collision frequency between $i$ and $j\left[\mathrm{~m}^{3} \mathrm{~s}^{-1}\right]$ \\
\hline$e$ & electron charge $[C]$ & $\chi$ & susceptibility [ ] \\
\hline$F_{\text {mag }}$ & magnetic force $[N]$ & $\kappa$ & reciprocal DEBYE length $\left[\mathrm{m}^{-1}\right]$ \\
\hline$H$ & magnetic field strength $\left[\mathrm{A} \mathrm{m}^{-1}\right]$ & $\lambda$ & wavelength [m] \\
\hline$h$ & absolute surface to surface distance $[\mathrm{m}]$ & $\mu_{0}$ & permeability constant of vacuum $\left[N A^{-2}\right]$ \\
\hline$I$ & ionic strength $\left[\frac{\mathrm{mol}}{\mathrm{m}^{3}}\right]$ & $\Phi_{v, N M}$ & volume concentration ratio non magnetic particles \\
\hline$k$ & Boltzmann constant $\left[\mathrm{J} \mathrm{K}^{-1}\right]$ & & 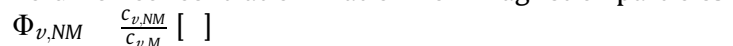 \\
\hline$M$ & magnetization $\left[\mathrm{A} \mathrm{m}^{-1}\right]$ & $\varphi$ & surface potential [V] \\
\hline$M_{\text {sat }}$ & Saturation magnetization $\left[\mathrm{A} \mathrm{m}^{-1}\right]$ & $\Psi_{e l}$ & electrostatic interaction potential [J] \\
\hline$N$ & number concentration $\left[\mathrm{m}^{-3}\right]$ & $\Psi_{v d W}$ & Van der Waals interaction potential [J] \\
\hline$N_{A}$ & AVOGADRO number $\left[\mathrm{mol}^{-1}\right]$ & $\rho$ & density $\left[\frac{\mathrm{kg}}{\mathrm{m}}\right]$ \\
\hline & rotary speed $\left[\mathrm{s}^{-1}\right]$ & $\Theta$ & contact angle $\left[{ }^{\circ}\right]$ \\
\hline & & $\zeta$ & zeta potential $[\mathrm{V}]$ \\
\hline
\end{tabular}

1955 , in which they euphorically welcomed a new age of the throwaway society that promised dramatic time savings and life improvements through the introduction of single use goods in the consumer market (Luce, 1955). And in fact, plastic as a material is all around us in current everyday life, although the general perception in society has seemingly changed. The yearly report of PlasticsEurope states that in the year 2018 nearly 350 million met ric tons (MT) of plastic were produced worldwide (PlasticsEurope, 2018). Furthermore, 27.1 MT of plastic have been collected, while the usage in recycling and energy recovery are steadily out growing the declining landfill. However, comparing these numbers suggests that a large proportion of the produced plastic is not recovered and ends as litter in our environment. Jambeck et al. (2015) state that in the year 20104.812 .7 MT of plastic were transported into the oceans worldwide, with primary emitters being China and Indonesia. Sadly, these numbers become vivid in the growing number of pictures and media reports of whole plastic patches floating in the sea. This work is concerned with so called microplastics, a term that is often used in the context of aquatic plastic litter, but still not consistently defined in the literature. The categorization used in this work is given by Andrady (2015) and defines plastic particles $>1 \mathrm{~mm}$ as mesoplastics, particles $<1 \mathrm{~mm}$ as microplastics and in accordance with (Klaine et al., 2012) particles $<100 \mathrm{~nm}$ as nanoplastics. It is important to emphasize the correct terminology since the size of plastic debris often regulates the extent of hazard posed by these particles. Microplastics (and nanoplastics alike) are further classified into primary and secondary microplastics depending on their origina tion (Cole et al., 2011; Browne, 2015): Primary microplastics are specifically manufactured and used in their small size. Typical examples are micro beads in facial cleaners and cosmetics or syn thetic clothing fibers. In contrast, secondary microplastics are defined as small plastic fragments resulting from a breakdown or weathering process of larger plastic debris. Hereby, the main degradation principles are UV induced photodegradation in gen eral and thermo oxidation on beaches (Andrady, 2015). As a conse quence of both pathways combined, the total amount of microplastics measured in the sea is constantly increasing, while it appears that the mean particle sizes are declining (Cole et al.,
2011; Barnes et al., 2009; Galgani et al., 2015). Marine Litter poses various, more or less understood hazards upon wildlife: 557 spe cies have been reportedly affected by physical obstruction like entanglement or smothering of plastics (Kühn et al., 2015). How ever, one of the main concerns and threats lies in the possible ingestion of micro and nanoplastics by all different sizes of marine organisms resulting in an introduction into the food web. Microplastics are known to transport a conglomerate of various POPs (persistent organic pollutants), chemicals and heavy metals on their surface which are likely to be released upon ingestion with hard to estimate consequences (Rochman et al., 2013; Rochman, 2015). Although model calculations estimate that this pathway is unlikely to be of major importance (Gouin et al., 2011), it is undoubtedly a reason for major concern that needs addressing especially since many long term consequences remain unknown.

In order to reduce the emissions of microplastics into the envi ronment, effective processes for separating these fine polymeric particles from industrial and municipal wastewater streams are necessary. However, it seems that most research focuses on the characterization and quantification of microplastics and their potential hazards. While these investigations are of major impor tance, the search for feasible separation techniques from large water streams appears to be subordinate. An effective separation is particularly challenging due to two aspects: (1) Large water vol umes with low solids concentrations and (2) small particle sizes in the lower to sub micron range. Fig. 1 shows the spectrum of well implemented filtration and sedimentation techniques dependent on their most common particle sizes as well as process scales. Note, that this graph is meant to give a qualitative categorization and a general overview of the field rather than exact boundaries for the presented separation processes. Fig. 1 displays the general trend that with decreasing particle size, the process scale and treated water volumes are also decreasing. Furthermore, it is important to note that sedimentation apparatuses generally struggle when the density difference between solid and liquid phase is not signif icant which further limits their possibilities for many low density plastics. Cake filtration is difficult in highly dilute suspensions as no filter cake can be generated and thus separation efficiencies suffer. Depth filtration constitutes an exception to the above 


\section{particle size / $\mu m$}

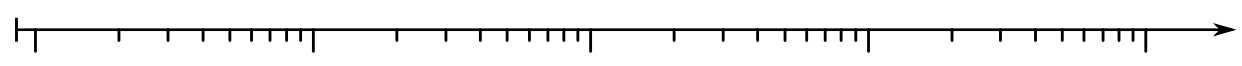

$\begin{array}{lllll}0.01 & 0.1 & 1 & 10 & 100\end{array}$

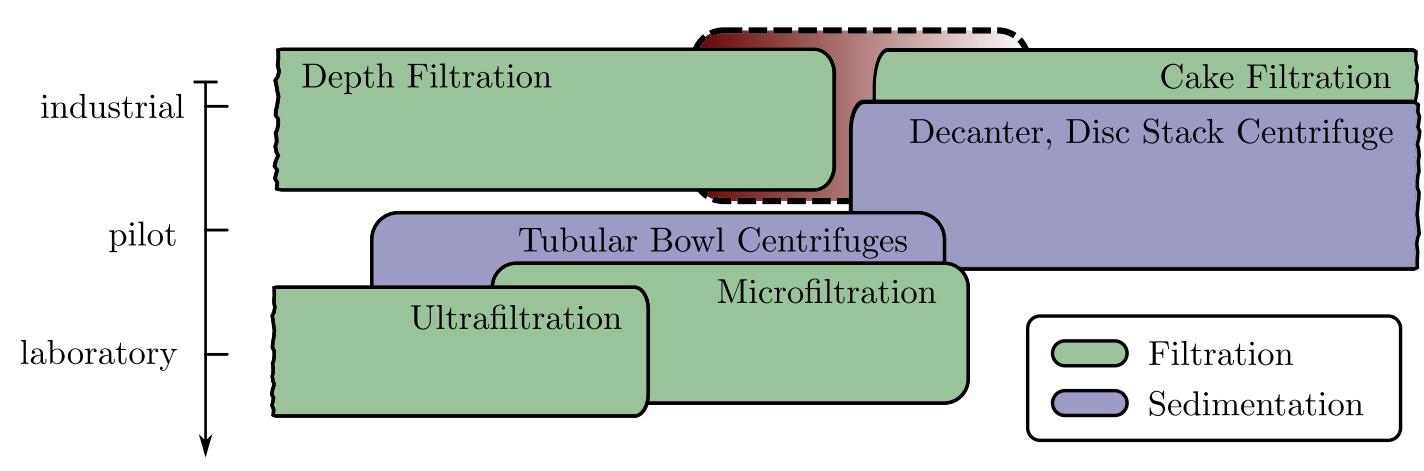

process scale

Fig. 1. Non exhaustive spectrum of well-implemented filtration and sedimentation techniques (Spelter et al., 2010; Ladislav, 2000). The dashed box indicates some sort of separation gap.

mentioned trend, as it is able to cover large water volumes, small particle sizes and is most efficient in dilute suspensions. These qualities make depth filtration generally an interesting technique in microplastic separation, although there remain some disadvan tages as well: Mainly the overall size of depth filtration appara tuses as well as the additional flow resistance resulting from the pressure drop represent the main weaknesses of this process. Fig. 1 further shows, that especially in the lower to sub micron range some sort of separation gap arises (dashed box) in which industrial scale separation is challenging. All of these facts com bined indicate that for this demanding separation task, it may be rewarding to look into newer and less known separation tech niques. The given illustration is by no means exhaustive and espe cially in the sub micron range many other, often overlooked separation processes offer distinct advantages which are summa rized in Wu et al. (2013). Among others, chemical coagulation, flotation and magnetic seeded filtration are discussed as possible separation methods. Coagulation and flotation are already used in wastewater treatment plants and especially flotation may offer distinct benefits in the separation of microplastics due to their often hydrophobic surfaces.

This work focuses on magnetic seeded filtration (MSF) and investigates its applicability as well as its obstacles in the separa tion of microplastics. Generally, the process can be divided into two steps: First, magnetic seed particles are dispersed into the sus pension and agglomerate with the target particles (microplastics). Then, these newly formed hetero agglomerates can be removed by magnetic separation due to the magnetic properties of the seed particles. At first, this process may seem effortful and costly, how ever by choosing the right magnetic seed particles as well as using permanent magnets MSF can be an efficient separation process. Furthermore, due to the strong magnetic force separation matrices in magnetic separation are generally designed with a much lower flow resistance and pressure drop compared to classical filtration techniques. Another major benefit of MSF is that its size depen dency can be manipulated by the choice of magnetic seed particles, not limiting this process to a certain minimum particles size. To this day, MSF has been implemented in the treatment of chemical mechanical polishing (Chin et al., 2006) as well as backside grind ing (Wan et al., 2011) wastewater and achieved excellent removal efficiencies when seed and target particles were oppositely charged. Electrostatic interactions also presented themselves as the driving force in the separation of organic dyes in wastewater (Fang et al., 2010). Furthermore, multiple studies showed that the removal of heavy metals can be accomplished in an efficient manner by MSF (Terashima et al., 1986; Karapinar, 2003). Even the implementation for large scale treatment of highly turbid water was studied and showed promising results (Chin and Fan, 2010). Thus, MSF may indeed be a suitable process for the separa tion of microplastics, although certain concerns regarding separa tion efficiencies, process costs and scale up potential are valid and need to be overcome. This study explicitly aims to examine the viability of MSF in the separation of microplastics on a labora tory scale and may therefore yield valuable information about a possible, future large scale implementation.

\section{Materials \& methods}

\subsection{Magnetic seeded filtration theory}

As mentioned above, the MSF process can be divided in a hetero agglomeration step followed by a magnetic separation. In the following section, the basic theory concerning both of these steps is given which will allow for a thorough discussion of the experimental results.

The reduced agglomeration kinetic given in Eq. (1) is a good starting point to discuss the relevant parameters for the hetero agglomeration step. Consider a suspension containing particles of type $i$ and particles of type $j$ that agglomerate to form $i j$. Under the assumption that only two body collisions occur, the rate of for mation of $i j$ can be written as in Eq. (1). Note that higher order col lisions are usually ignored as they only become relevant at very high particle concentrations (Elimelech, 1998).

$\frac{d N_{i j}}{d t} \propto N_{i} N_{j} \beta_{i, j} \alpha_{i, j}$

$N_{i}$ and $N_{j}$ are the number concentrations of agglomerating particles, $N_{i j}$ is the number concentration of a resulting agglomerate, $\beta_{i, j}$ is the collision frequency and $\alpha_{i, j}$ is the collision efficiency between $i$ and $j$. Both $\beta$ and $\alpha$ have a rather graphic meaning for the process: The col lision frequency describes the probability that two agglomeration partners $i$ and $j$ collide and is thus mainly dependent on the flow conditions in the system. The collision efficiency on the other hand specifies the probability that both partners agglomerate upon 
collision and can be estimated from the interface potential between them. In the scope of this work the so called DLVO (van der Waals and electrostatic) interactions are of utmost relevance. Eq. (2) gives a simple expression for the van der Waals potential, while Eq. (3a) further defines the electrostatic potential for the case of two inter acting spheres (Elimelech, 1998).

$$
\Psi_{v d W, i j} \frac{A_{H} r_{i} r_{j}}{6 h\left(r_{i}+r_{j}\right)}
$$

$$
\Psi_{e l, i j} \frac{128 \pi r_{i} r_{j} I N_{a} k T}{\left(r_{i}+r_{j}\right) \kappa^{2}} \gamma_{i} \gamma_{j} \exp (\kappa h)
$$

$$
\kappa \quad \sqrt{\frac{2 e^{2} I N_{a}}{\epsilon k T}} \mid \gamma_{i} \tanh \left(\frac{e \varphi_{i}}{4 k T}\right)
$$

Here, $A_{H}$ is the HAMAKER constant, $r_{i}$ the radius of particle $i$ and $h$ rep resents the absolute surface to surface distance. Further, $I$ is the ionic strength, $N_{a}$ the AVOGADRo number, $e$ the electron charge, $\kappa$ the reciprocal DEBYE length which can be calculated by Eq. (3b) and $\varphi$ is the surface potential of the particles. However, the surface potential is not measurable directly and thus the zeta potential $\zeta$ is mainly used as an approximation. Besides giving a general under standing for the governing process parameters, Eq. (1) (in its full form) can be used in combination with population balances to model agglomeration processes. This was performed by many authors (also of this work) (Tsouris et al., 1995; Chin et al., 1998; Taboada Serrano et al., 2005; Rhein et al., 2019) and will not be dis cussed further here.

The second process step is concerned with the separation of the newly formed hetero agglomerates. Thereby, high gradient mag netic separation (HGMS) offers an effective and (with implementa tion of permanent magnets) energy efficient solution. The magnetic force on agglomerates as the driving separation mecha nism can be described by Eq. (4) (Svoboda, 2004)

$F_{\text {mag }} \quad \mu_{0} V_{p, M} M \nabla H$

where $\mu_{0}$ is the permeability constant of the vacuum, $\nabla H$ the gra dient of magnetic field strength, $V_{p, M}$ the total volume of magnetic particles in an agglomerate and $M$ represents their magnetization. Eq. (4) assumes strongly magnetic particles (negligible magnetiza tion of the fluid), that only magnetic particles contribute to the sep aration and further, in a sufficiently strong field, the magnetization $M$ can be considered a material constant, namely the saturation magnetization $M_{\text {sat }}$. Both of these assumptions are generally appli cable in MSF which leaves the main dependencies to be the agglom erate volume $\left(\propto r_{i}^{3}\right)$ and the field strength gradient. In order to achieve a high field strength gradient, a strong magnetic flux den sity in the typical range of $B \quad 0.2 T \quad 0.5 T$ is necessary (Menzel et al., 2013), which can be generated by an electromagnet or perma nent magnet. Since electromagnets require large amounts of energy and cooling, an openable Halbach permanent magnet that yields field strengths suitable for HGMS applications (up to 0.37T) and does not demand further energy input was constructed in previous
(Menzel et al., 2013) and is used in present work. However, a strong magnetic field alone is not sufficient: In order to generate gradients in the process volume, a separation matrix consisting of fine ferro magnetic wires needs to be introduced that attracts and collects magnetic particles and agglomerates. Here, (Svoboda, 2004) states that the gradient of the field is inversely proportional to the wire diameter $\left(\nabla H \propto 1 / a_{\text {wire }}\right)$ and therefore the separation efficiency is maximized by using as fine a matrix as possible.

\subsection{Experimental studies}

As mentioned above, the term microplastic encompasses basi cally every type of polymer particle with a mean diameter of $<1 \mathrm{~mm}$. However, especially particles in the lower to sub micron range are of interest in this paper since they are challenging to remove by classical methods and further exhibit special hazards due to possible ingestion. As representatives of this class, polyvinylchloride (PVC) as well as polymethylmethacrylate (PMMA) were selected and used in the experiments, while mag netite $\left(\mathrm{Fe}_{3} \mathrm{O}_{4}\right)$ particles were used as magnetic seeds. PVC has been shown to carry a disproportionate number of hazardous chemical products and is one of the most used industrial polymers (PlasticsEurope, 2018; Lithner et al., 2011). While the market share of PMMA is rather small, it is known to be used in cosmetics and can thus be seen as relevant in the field of primary microplastics. Furthermore, mineral magnetite is used as magnetic seed particles, primarily because it is cheap and non toxic, which will allow for a later use in larger process scales. Table 1 summarizes the main properties of the material systems used. It is important to note, that all particle systems are polydisperse in order to further repre sent real world separation problems. Another crucial material property for agglomeration processes is the zeta potential of these particle systems, which is plotted over the $\mathrm{pH}$ in Fig. 2. These mea sured values are in good agreement with known zeta potential val ues from the literature (Sun et al., 1998; Kirby and Hasselbrink, 2004).

The entire experiment can be split into four process steps which are described in the following section. Before conducting the actual agglomeration, the suspension is prepared by means of ultrasonic dispersion. First, the given amount of non magnetic particles is added to $0.5 \mathrm{~L}$ of ultrapure water with a residual ionic strength of $I \approx 10{ }^{6} \mathrm{~mol} / \mathrm{L}$. Then, the ionic strength is adjusted by adding $2 \mathrm{~mol} / \mathrm{L}$ analytic grade sodium chloride $(\mathrm{NaCl})$ solution, while the $\mathrm{pH}$ is regulated with $0.5 \mathrm{~mol} / \mathrm{L}$ hydrochloric acid $(\mathrm{HCl})$ and sodium hydroxide $(\mathrm{NaOH})$. The suspension is subsequently dispersed with the Digital Sonifier 450 (Branson) for $20 \mathrm{~min}$ in order to break up existing non magnetic agglomerates. After the first dispersion step a sample $\left(S_{0}\right)$ of this suspension is taken and stabilized with $0.5 \mathrm{~g} / \mathrm{L}$ tetrasodium pyrophosphate in order to hinder agglomeration before the measurement of this sample. Next, the magnetic parti cles are added and the suspension is further dispersed for $10 \mathrm{~min}$. After the dispersion step, the prepared suspension is added to the agglomeration cell and agitated at the respective rotary

\begin{tabular}{|c|c|c|c|}
\hline & Magnetic (M) & Non-magnetic (NM) & \\
\hline Material & Magnetite $\left(\mathrm{Fe}_{3} \mathrm{O}_{4}\right)$ & Polyvinylchloride (PVC) & Polymethylmeth-acrylate (PMMA) \\
\hline Manufacturer & Kremer Pigments, Germany & Vinnolit, Germany & Goodfellow, Germany \\
\hline Mean diameter $x_{50,3}$ & $2.75^{\mathrm{a}} \mu \mathrm{m}$ & $2.06^{\mathrm{a}} \mu \mathrm{m}$ & $5.98^{\mathrm{a}} \mu \mathrm{m}$ \\
\hline Density $\rho$ & $5200 \frac{\mathrm{kg}}{\mathrm{m}^{3}}$ & $1400 \frac{\mathrm{kg}}{\mathrm{m}^{3}}$ & $1190 \frac{\mathrm{kg}}{\mathrm{m}^{3}}$ \\
\hline Saturation magnetization $M_{s a t}$ & $4.94 \cdot 10^{5} \frac{\mathrm{A}}{\mathrm{m}}\left(\mathrm{H} \quad 1.75 \cdot 10^{5} \frac{\mathrm{A}}{\mathrm{m}}\right)$ & - & - \\
\hline Contact angle air,water $\Theta$ & $0^{\circ \mathrm{b}}$ & $91.7^{\circ \mathrm{b}} \pm 4.1^{\circ}$ & $21.4^{\circ \mathrm{b}} \pm 4.0^{\circ}$ \\
\hline
\end{tabular}

Table 1

Particle systems used in experimental studies.

a Particle size distributions and therefore mean diameters were measured with laser diffraction (HELOS/QUIXEL, SympaTec GmbH, Germany).

b Contact angles were measured by sessile drop method. Samples were prepared in a hydraulic press with constant force (15 kN). 


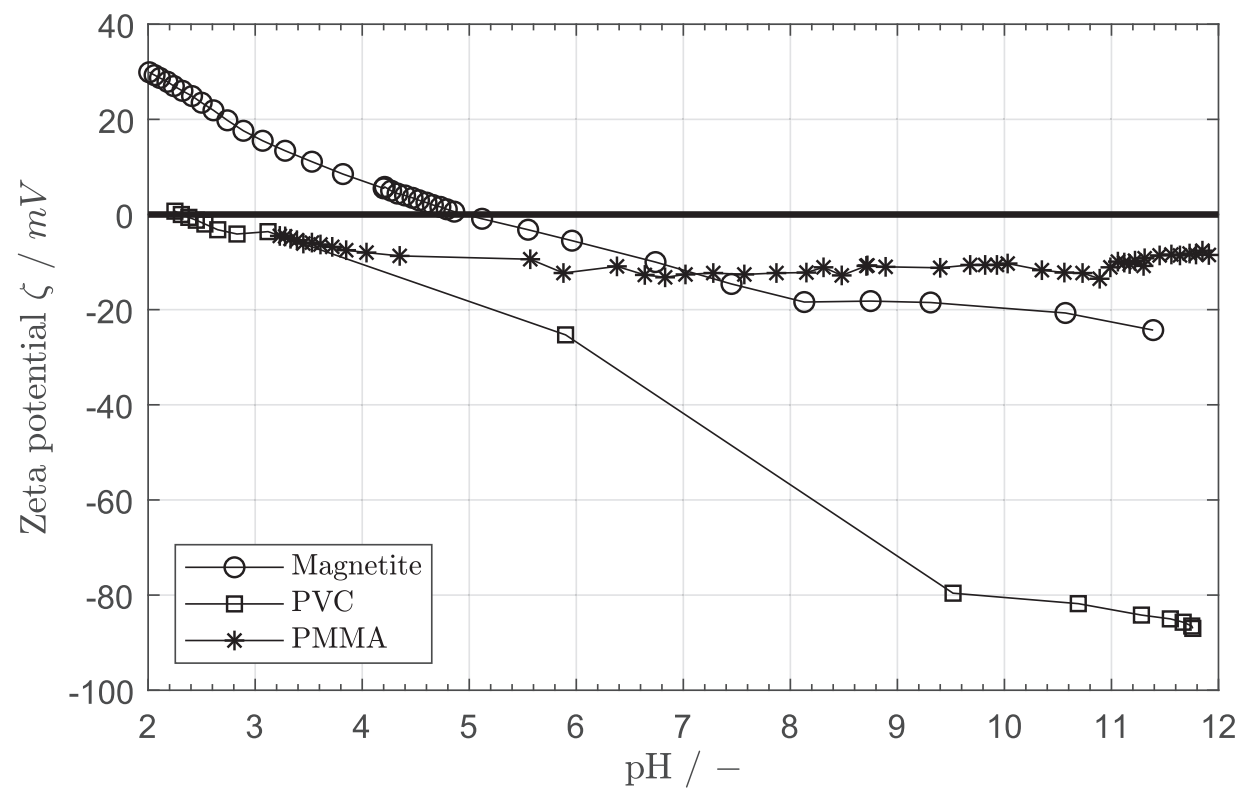

Fig. 2. Zeta potential over pH for used particle systems.

speed $n$ for the agglomeration time $t_{A}$ in order to induce an agglomeration of the particles. Following the agglomeration pro cess, the separation of the non agglomerated magnetic primary particles as well as hetero agglomerates is initiated. Therefore, an iron matrix is lowered into the suspension and the whole tank is brought into the magnetic field produced by the openable Halbach permanent magnet described above $\left(\begin{array}{ll}B & 0.37 T\end{array}\right)$. Due to the high gradients produced by the fine ferromagnetic mesh, the magnetic particles as well as hetero agglomerates are collected.The separa tion is performed at constant rotary speed $n_{\text {sep }} \quad 100 \mathrm{~min}{ }^{1}$. In pre liminary tests, a nearly instant and complete separation of the magnetic particles could be detected, however in order to also guarantee total separation of hetero agglomerates the separation time was increased to $t_{\text {sep }} 5 \mathrm{~min}$. Fig. 3 shows the schematics of the agglomeration cell and the two states for agglomeration and separation. The main difference between the two states lies in the position of both agglomeration cell and separation matrix: For separation the entire cell is placed in a magnetic field and the separation matrix is lowered into the suspension by hand in order to induce magnetic separation. A four bladed $45^{\circ}$ blade tur bine is used as agitator that yields an approximate shear rate of $G \approx 300 \mathrm{~s}^{1}$ at mean rotary speed $n 200 \mathrm{~min}^{1}$ (Chin et al.,
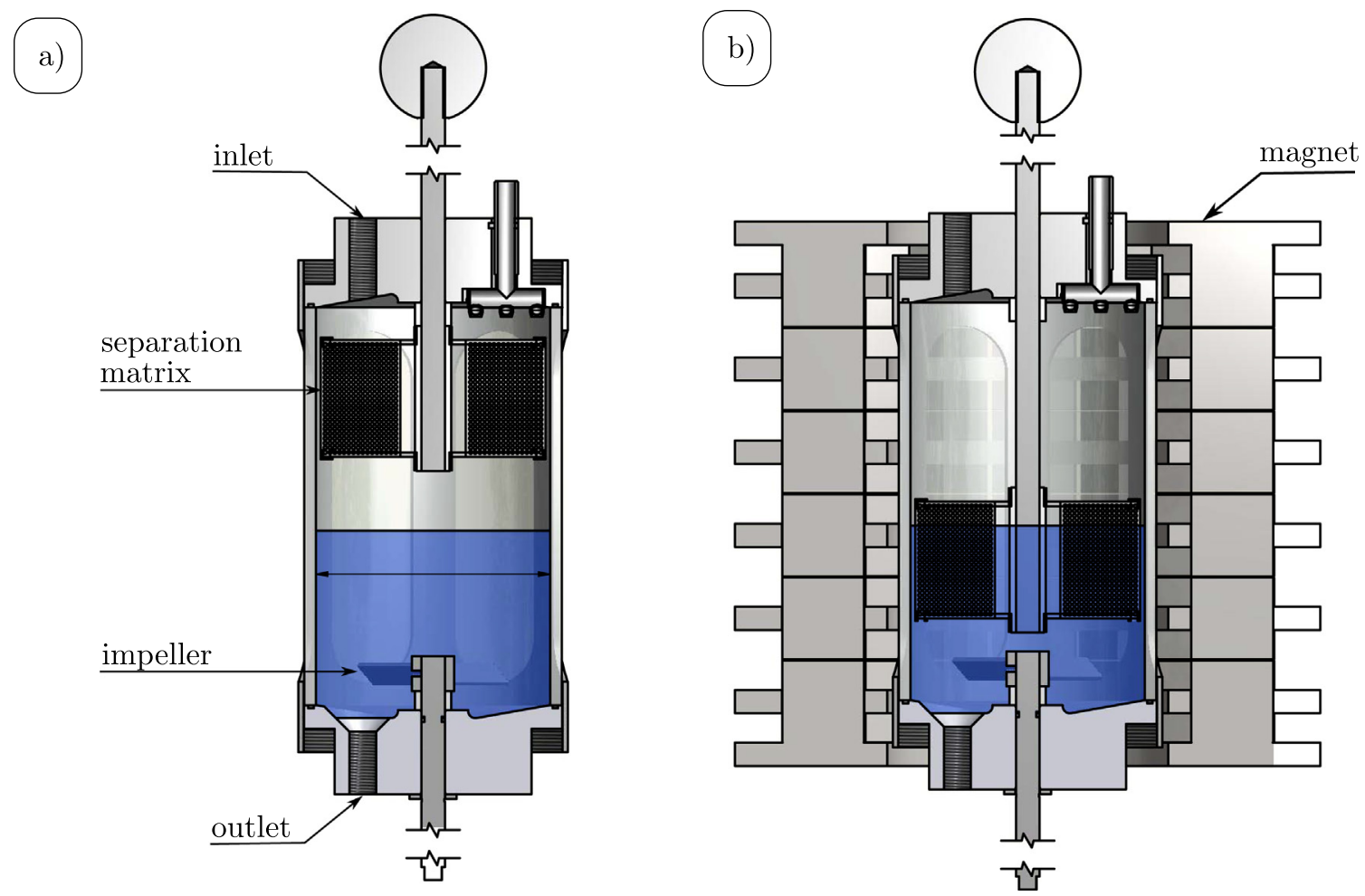

Fig. 3. Agglomeration cell and its two states: (a) agglomeration, (b) separation. 
1998). Subsequently to the HGMS, the suspension, which now only contains the unseparated particles and agglomerates, is collected and must be treated before measurement. Therefore, the suspen sion is again dispersed with the sonifier for $10 \mathrm{~min}$ and stabilized with tetrasodium pyrophosphate. Then, a sample of the dispersed residual suspension is taken $\left(S_{i}\right)$ and analyzed together with the $S_{0}$ sample by means of absorbance spectroscopy at $\lambda \quad 600 \mathrm{~nm}$. The validity of the Beer Lambert law was ensured in previous stud ies, so that the absorbance value can be transferred into a concen tration value. Under the assumption that all magnetic particles as well as all hetero agglomerates (agglomerates containing mag netic particles) are separated in the HGMS step, a separation effi ciency for the non magnetic particles $T_{N M}$ can be defined according to Eq. (5).

$$
\begin{aligned}
& T_{N M}(t) \quad\left(\begin{array}{ll}
1 & \left.\frac{A_{600}(t)}{A_{600}(t} \quad 0\right)
\end{array}\right) \cdot 100 \% \\
& \left(\begin{array}{ll}
1 & \left.\frac{c_{v, N M}(t)}{c_{v, N M}(t} 0\right)
\end{array}\right) \cdot 100 \%
\end{aligned}
$$

Here, $A_{600}(t)$ represents the absorbance values at wavelength $\lambda \quad 600 \mathrm{~nm}$ and $c_{v, N M}(t)$ represents the volume concentration of nonmagnetic particles in the suspension at process time $t$.

\section{Results \& discussion}

Before conducting any experiments, a proof of principle has to be established. For this purpose, hetero agglomerates produced in the agglomeration cell were collected and investigated under a SEM microscope. An example for such a recording is given in Fig. 4. It becomes apparent, that a hetero agglomeration process has indeed taken place and the principle can thus be regarded as proven.

However, SEM images only yield qualitative information and therefore, quantitative investigations are conducted and presented below. First, a system analysis has to be performed in order to identify all possibly relevant parameters. The factors that appear to be process determining then need to be assigned with realistic values. Table 2 shows the process parameters as well as their respective values. For now, only the default values are of interest, while the and + values will be considered later. Since the electro static interactions are known to be of utmost importance, the first study was an investigation of the separation efficiency dependent on the $\mathrm{pH}$ value. Therefore, all parameters were set to their default value, while the $\mathrm{pH}$ was varied. The results are shown in Fig. 5. First of all, high separation efficiencies of up to $T_{N M} \approx 95 \%$ could be achieved for both particle systems although the concentration val ues of both magnetic and non magnetic particles are relatively low $\left(\approx 10^{2} \mathrm{vol} \%\right.$ ). It can be seen that a clear relationship between $\mathrm{pH}$ and $T_{N M}$ exists for PVC particles: At low $\mathrm{pH}$ values, the separation efficiency is high and relatively constant. At around $p H$ 6, the separation efficiency decreases until nearly no separation is achieved at $p H \quad 11$. This effect can be explained through a look at Fig. 2: Magnetite exhibits a point of zero charge (pzc) at $p H \approx 5$ while being positively charged for lower and negatively charged for higher $p H$ values. Concurrently, PVC is negatively charged throughout the whole $\mathrm{pH}$ range with increasing absolute values for larger $\mathrm{pH}$. This results in low repulsive and even attrac tive electrostatic interactions between the magnetic seed particles and the non magnetic target particles for $\mathrm{pH}<6$ which ultimately results in high separation efficiencies. With increasing $\mathrm{pH}$, the neg ative surface charge of magnetite increases which leads to a drop in agglomeration and thus separation efficiency. It should be high lighted however, that in the broader neutral $p H$ range between 5 and 9 high efficiencies are easily realizable. Furthermore, the high separation efficiency at $p H \quad 6$ indicates that it is sufficient to reduce the electrostatic repulsion and not necessary to evoke fur ther attraction between the particle systems, as at this point mag netite is nearly uncharged and yet high separation efficiencies are achieved. This fact is especially interesting when it comes to esti mating process results for other, non polar and thus non chargeable polymer systems as for example polyethylene (PE): Since they will not exhibit electrostatic repulsion, their separation result can be estimated as high. Regarding PMMA, a constantly high separation efficiency could be measured throughout the whole range of $\mathrm{pH}$ values. Considering Fig. 2, it is apparent that

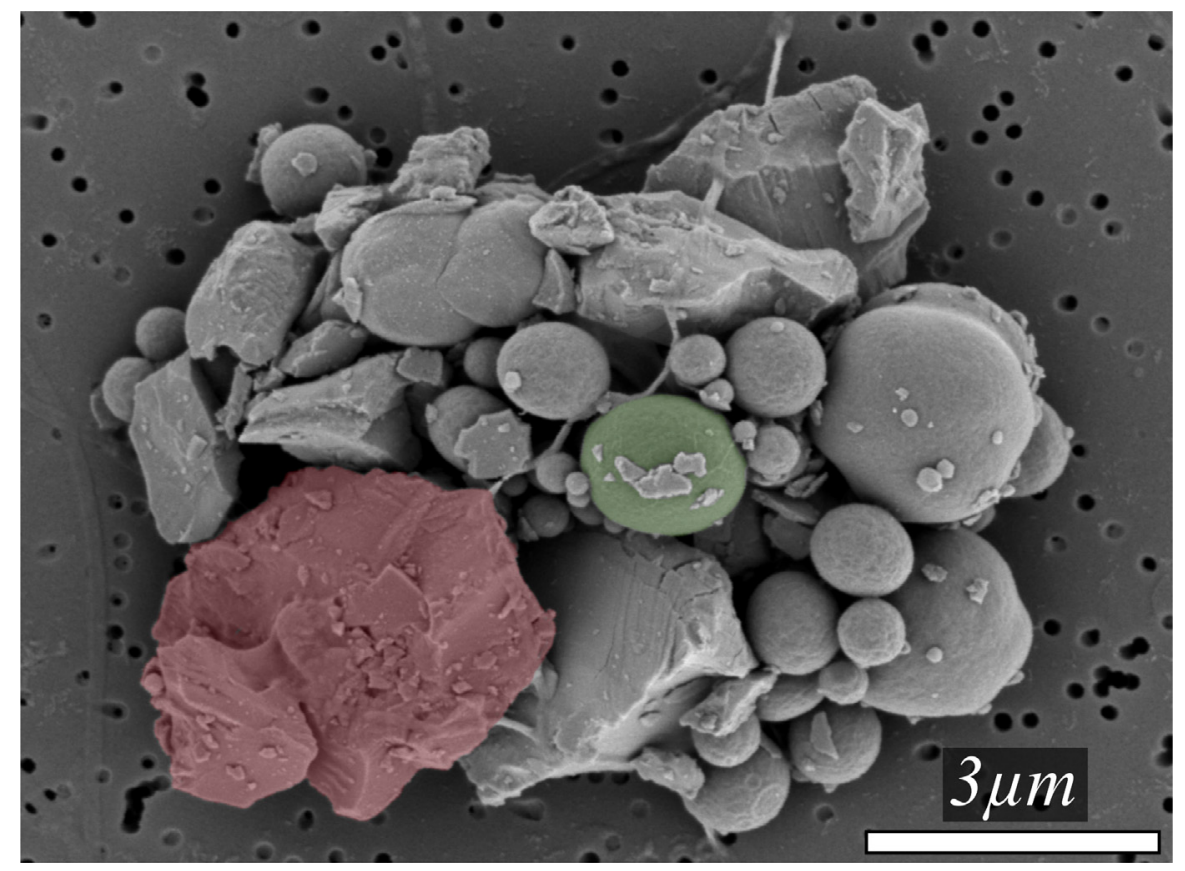

Fig. 4. SEM image of a collected hetero-agglomerate. The angular particles are magnetite (colored red as an example), while the spherical particles are PVC (colored green as an example). (For interpretation of the references to colour in this figure legend, the reader is referred to the web version of this article.) 
Table 2

Process relevant parameters and their defined levels.

\begin{tabular}{|c|c|c|c|c|c|c|}
\hline \multirow[b]{2}{*}{ Name } & \multirow[b]{2}{*}{ Description } & & \multirow[b]{2}{*}{ Unit } & \multicolumn{3}{|c|}{ Factor level } \\
\hline & & & & Default & - & + \\
\hline$n$ & Rotary speed & & {$\left[\min ^{1}\right]$} & 200 & 150 & 250 \\
\hline$c_{v, M}$ & Volume concentration magnetic particles & & [vol\%] & 0.0075 & 0.005 & 0.01 \\
\hline$\Phi_{\nu, N M}$ & Volume concentration ratio non-magnetic particles $\Phi_{v, N M}$ & $\frac{c_{v, N M}}{c_{\nu, M}}$ & {$[-]$} & 1 & 0.5 & 2 \\
\hline$I$ & Ionic strength & & {$\left[\frac{\mathrm{mol}}{\mathrm{L}}\right]$} & $10^{2}$ & $10^{3}$ & $10^{1}$ \\
\hline$p H$ & $\mathrm{pH}$ value & & {$[-]$} & 9 & 6.4 & 11 \\
\hline$t_{A}$ & Agglomeration time & & {$[\mathrm{min}]$} & 10 & - & - \\
\hline
\end{tabular}

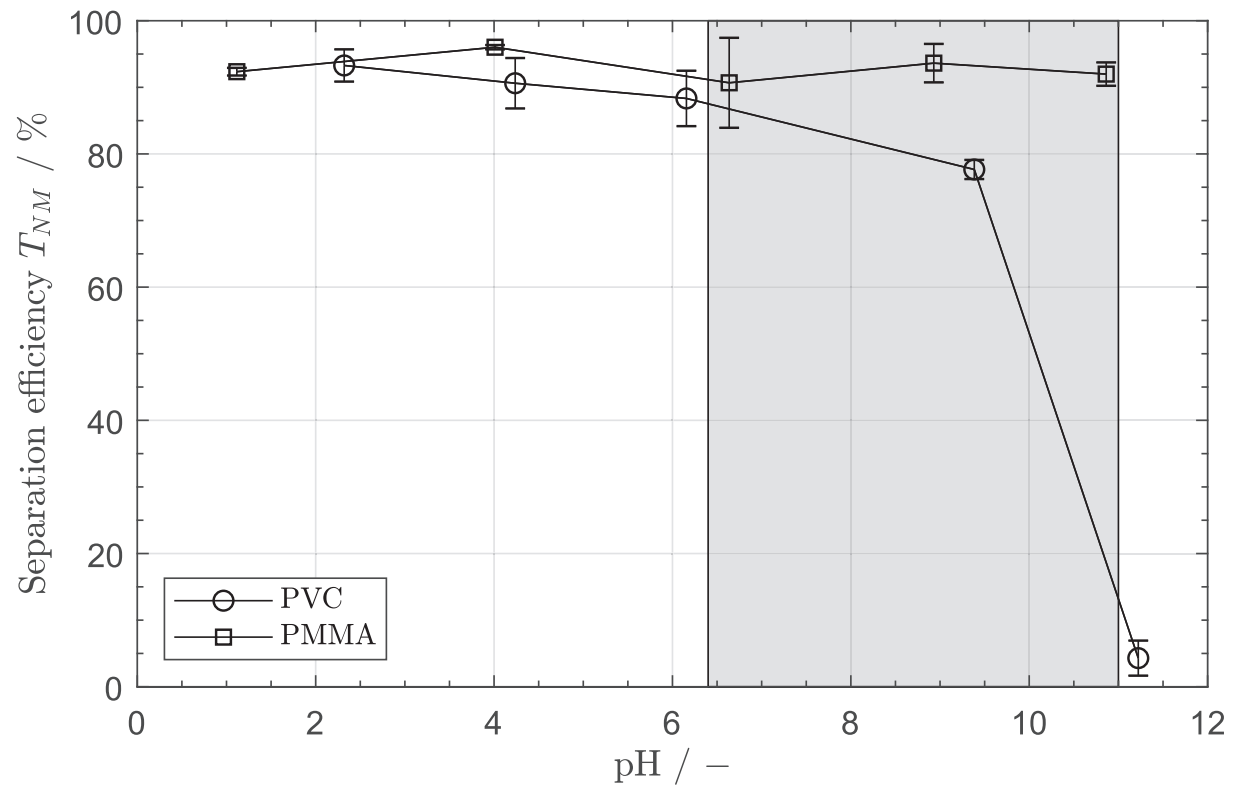

Fig. 5. Separation efficiency over pH for default process parameter combination (see Table 2).

the PMMA particles exhibit a small negative zeta potential of $\zeta \approx 10 \mathrm{mV}$ that is independent of the $\mathrm{pH}$ value. This results in a small electrostatic repulsion even for negatively charged magnetite particles at higher $\mathrm{pH}$ values and explains the constantly high sep aration efficiencies.

In a subsequent step, the range $6.4<p H<11$ was examined more closely in order to determine whether it is possible to force hetero agglomeration between the particles despite them having the same charge. This area is highlighted in Fig. 5 by the shaded area. Since PMMA already exhibits high separation efficiencies, only PVC particles are studied. A design of experiment (DOE) was conducted to investigate the influence of the process parameters on the separation efficiency. Five parameters (factors) are varied on two levels, while the agglomeration time is held constant at $t_{A} 10 \mathrm{~min}$. The lower ( ) and higher $(+)$ level values of the respective factors are given in the two rightmost columns in Table 2. A full factorial DOE of given problem with $l \quad 5$ yields $k \quad 2^{l} \quad 32$ parameter combinations that have to be conducted by experiment. However, a fractional factorial DOE can be used to lower the amount of experiments to $k \quad 2^{l 1} \quad 16$. The $2^{51}$ design still allows a clean resolution of all main and two way inter action terms, since they are only aliased with interactions of an order larger than two (Berger, 2018). The separation efficiencies for all 16 process parameter combinations were determined in triplicate and the mean separation efficiencies as well as the stan dard deviations were calculated. These values were then used in a multiple regression calculation in order to determine the effects of all factors and two way interactions. A linear model as shown in
Eq. (6) was used. The $X$ variables thereby represent the factors given in Table 2 normalized to their varied range. Due to the nor malization, the coefficients $E_{i}$ can be understood as the effects of the respective factors on the separation efficiency and can be com pared directly.

$T_{N M} \quad \sum_{i}^{l} E_{i} X_{i}+\sum_{i}^{l} \sum_{j}^{l} \sum_{i+1}^{l} E_{i, j} X_{i} X_{j}$

The parameter study yielded separation efficiencies in the range of $3.95 \%<T_{N M}<96.02 \%$. As mentioned above 16 process param eter combinations were measured in triplicate and the average standard deviation for each combination was $\bar{\sigma} 2.78 \%$, which is considered reasonable. With the right set of process parameters, the separation efficiency for $p H 11$ could be improved to $T_{N M} \quad 51.74 \%$, which demonstrates that a thorough process opti mization may lead to a further improvement of the already promising results in Fig. 5. The absolute effects of the factors on the separation efficiency are shown in Fig. 6. Again, it can be seen that the $p H$ has a strong negative effect on $T_{N M}$ meaning, that with decreasing $\mathrm{pH}$ value, the separation efficiency increases. This is due to the decreasing negative zeta potential of the magnetite particles and was discussed at length above. Further, the ionic strength $I$ exhibits a large positive effect on $T_{N M}$, meaning that the separation efficiency rises with increasing ion concentration. This effect can be explained by Eq. (3b): With increasing ionic strength, the recip rocal DEBYE length $\kappa$ increases which results in a decreasing range of the electrostatic repulsion (..exp $(\kappa t))$. Vividly described, this means that the loosely bound charges around a particle are com 


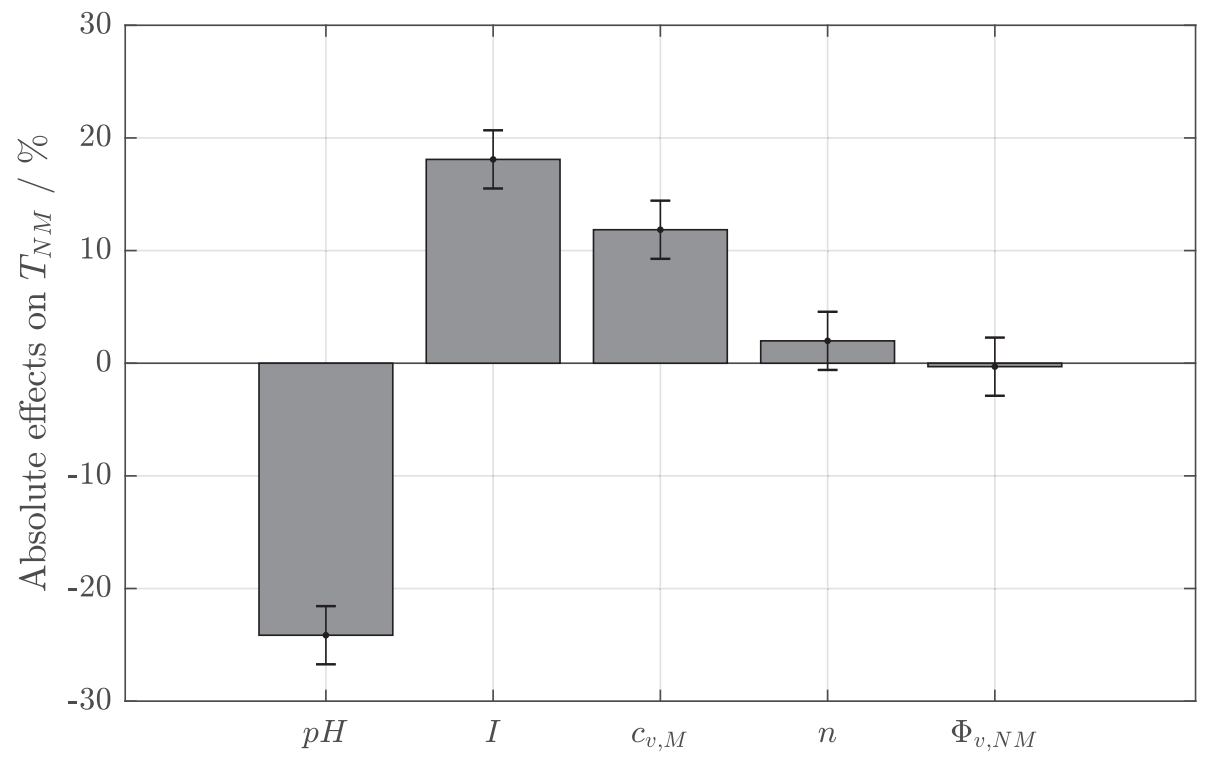

Fig. 6. Absolute effects of the varied factors on the separation efficiency.

pressed due to the high ion concentration resulting in a smaller ion shell and thus smaller interaction length scales. As third parame ter, the volume concentration of magnetic particles $c_{v, M}$ exhibits a positive effect on $T_{N M}$ which is not surprising regarding the basic agglomeration kinetic in Eq. (1): Increasing the amount of mag netic seed particles increases the absolute amount of NM M colli sion events and ultimately also the hetero agglomeration rates. However, the concentration ratio $\Phi_{v, N M}$ should follow the same logic and thus exhibit a negative effect on $T_{N M}$. This could not be shown in the experiments, where $\Phi_{v, N M}$ did not exhibit any signif icant effect. The same can be said about the rotary speed $n$, where no significant effect could be shown although a positive effect would have been expected due to an increasing $\beta_{i, j}$ value in Eq. (1). To sum up, most effects revealed by the multiple regression analysis show good agreement with the basic agglomeration the ory and highlight that especially the electrostatic interactions are governing the agglomeration process. However, such results are always dependent on the investigated range of the given factors, meaning that e.g. the rotary speed may indeed effect the system dramatically when investigated on a broader value range.

The results just discussed were all carried out for a constant agglomeration time of $t_{A} 10 \mathrm{~min}$, which suggests the question of whether further system optimization is also possible in this regard. Therefore, the agglomeration kinetics were measured for pH $\quad 4$ and $p H \quad 9$ and the results are shown in Fig. 7. Again, these studies are conducted with PVC, since PMMA is expected to show similar behavior and already yields optimal separation results. In order to further describe the kinetics mathematically, an analysis under simplifying assumptions starting from the reduced agglomeration kinetic given in Eq. (1) was conducted and is shown in Eq. (7).

$\frac{d N_{N M}}{d t} \quad N_{N M} \underbrace{N_{M} \beta_{N M, M} \alpha_{N M, M}}_{C_{1}} \quad N_{N M} C_{1}$

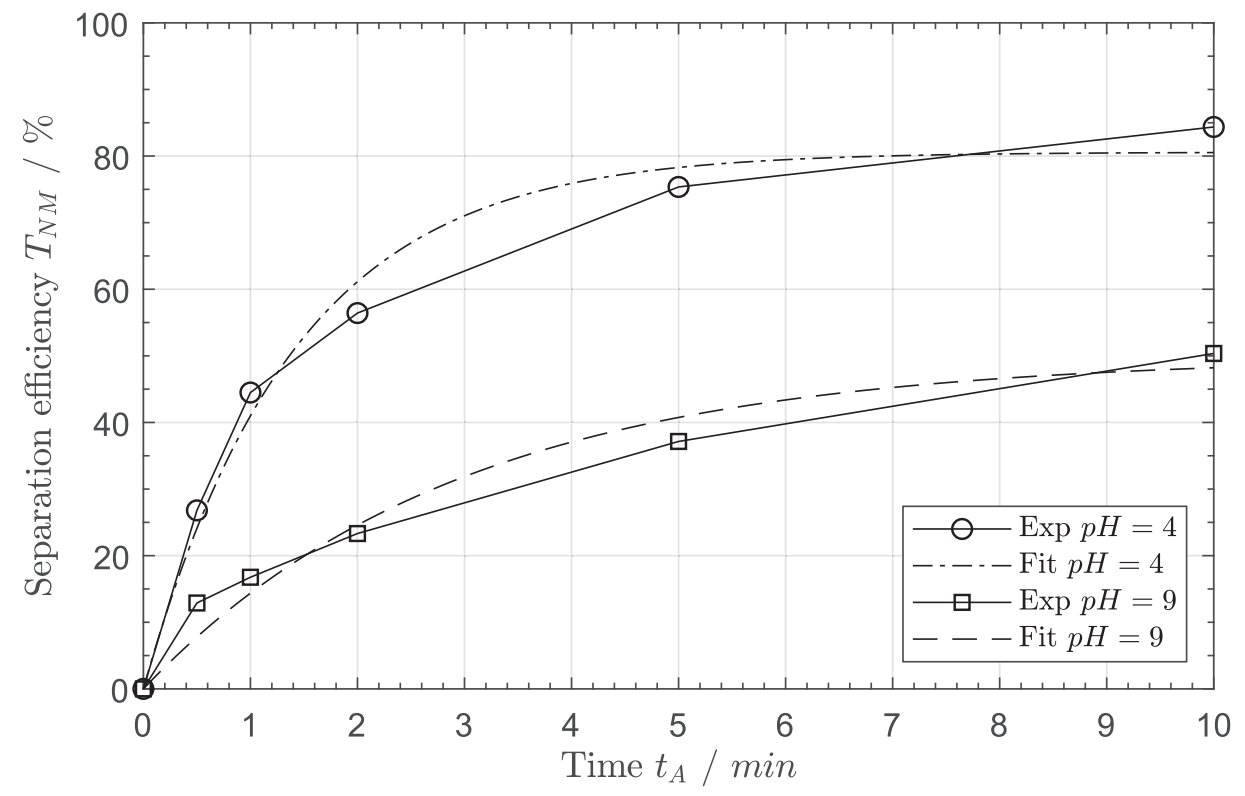

Fig. 7. Agglomeration kinetics and respective fits for PVC particles at $p H \quad 4$ and $p H \quad 9$. All other parameters are set on default value (see Table 2). 
Table 3

Fit parameters of Eq. (7c) on the measured kinetics for $p H \quad 4$ and $p H \quad 9$.

\begin{tabular}{llll}
\hline$p H$ & $C_{1}$ & $C_{2}$ & $R^{2}$ \\
\hline 4 & $0.7106 \min ^{1}$ & $80.59 \%$ & 0.9867 \\
9 & $0.3396 \min ^{1}$ & $49.88 \%$ & 0.9685 \\
\hline
\end{tabular}

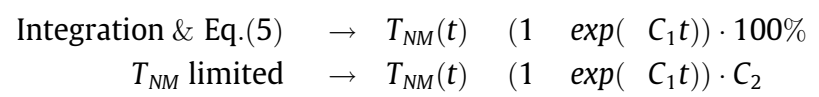

First, it is assumed that the change in non magnetic particles is only due to one agglomeration step with magnetic particles of known $\beta$ and $\alpha$ value. Further, a magnetic particle is presumed to be still available for subsequent agglomeration events after captur ing a non magnetic particle, which leads to the simplification $N_{M} \approx$ const. Thus, the product $N_{M} \alpha_{N M, M} \beta_{N M, M}$ can be combined into the agglomeration rate constant $C_{1}$ as shown in Eq. (7a). After inte gration, the term for $N_{N M}(t)$ can be inserted into Eq. (5) which yields the expression for the separation efficiency kinetic in Eq. (7b). However, the measured kinetics show a characteristic, limited growth which is why the factor $100 \%$ is converted into a limit con stant $C_{2}$ as shown in Eq. (7c). One explanation for this limitation lies in the fact that the average agglomerate size continues to grow steadily, while the absolute number of particles and agglomerates decreases. This leads to a reduction in the collision probability, which is not yet accounted for by the assumptions made above. Eq. (7c) was used to fit the measured values and the resulting kinetics are also plotted in Fig. 7 while the resulting constants and coefficients of determination $\left(R^{2}\right)$ are given in Table 3 . The apparent influence of the $p H$ on the separation efficiency demon strated in Fig. 5 can also be seen in the kinetics, as both the agglomeration rate constant $C_{1}$ and the limit constant $C_{2}$ are higher for lower $p H$ values. Further, it can be said that Eq. (7c) is able to represent the measured values well, as the $R^{2}$ values are close to 1 . For the purpose of process optimization, the measurement of agglomeration kinetics offers valuable information: Due to the lim ited exponential growth character, it is apparent that the process is most efficient at low agglomeration times, while the change in sep aration efficiency decreases for increasing run time. It is not possi ble to recommend an overall perfect agglomeration time, however after measuring the process kinetics and calculating the coeffi cients, the balancing of total separation efficiency and process time can be conducted on a profound basis.

\section{Conclusions}

This work shows that it is generally possible to separate fine polymeric particles from dilute suspensions in a highly efficient manner by magnetic seeded filtration. Separation efficiencies of $T_{N M} \approx 95 \%$ were achieved over a broad range of $p H$ values. Gener ally, the $p H$ and ultimately the surface charge of the particles showed to be the governing factors of the investigated hetero agglomeration process. However, a DOE in the range of higher $p H$ values, where both agglomeration partners exhibit an electrostatic repulsion, showed that high separation efficiencies could neverthe less be achieved by optimizing other process parameters such as the ionic strength or the magnetic seed concentration. From a pro cess engineering standpoint, it is important to maximize the agglomeration rate between magnetic seed and nonmagnetic target particles, which may be achieved by the following general guideli nes that can be conducted from the reduced kinetic in Eq. (1):

(i) Maximize attractive while minimizing repulsive interaction potentials between particle systems to increase $\alpha_{i, j}$. Here, primarily the variation of the surface potential through adjustment of the $p H$, but also high ion concentrations at repulsive potentials are purposeful.

(ii) Increase absolute particle number concentrations $\left(N_{N M} N_{M}\right)$. If the target concentration is given, increase the magnetic seed particle concentration.

(iii) Increase particle movement in order to increase $\beta_{i, j}$. Orthoki netic (flow controlled): Rise in energy dissipation/Re num ber. Perikinetic (diffusion controlled): Rise in temperature.

In addition, it was shown that it is useful to take a closer look at the process kinetics, since a further, time dependent optimization can be achieved here. By simplifying the general agglomeration kinetics, a model equation was found that was able to describe measured kinetics in an accurate manner. The coefficients obtained can then be used to make substantiated decisions on the balancing of total separation efficiency and total process time.

It is self evident that a number of fundamental questions remain unanswered regarding large scale implementation: Espe cially the recovery and regeneration of the magnetic seed particles will play an essential role. Further investigations are necessary, but this work shows that it is possible to counteract agglomeration by selecting suitable process parameters. Therefore, the agglomera tion process should generally be reversible if the suspension condi tions are specifically changed (e.g. by a $\mathrm{pH}$ shift).

In summary, this work demonstrates the basic viability of mag netic seeded filtration for the separation of microplastics. The here presented results and application notes may serve as a solid foun dation for future research that should be concerned with scale up issues of this process.

\section{Acknowledgment}

The authors acknowledge the support of the Institute for Tech nical Chemistry and Polymer Chemistry for help with the contact angle measurement probe preparation. The research project is funded by the DFG (German Research Foundation) in the priority program 2045 Highly specific and multidimensional fractionation of fine particle systems with technical relevance.

\section{References}

Andrady, A.L., 2015. Persistence of plastic litter in the oceans. In: Bergmann, M., Gutow, L., Klages, M. (Eds.), Marine Anthropogenic Litter. Springer International Publishing, pp. 57-72.

Barnes, D.K.A., Galgani, F., Thompson, R.C., Barlaz, M., 2009. Accumulation and fragmentation of plastic debris in global environments. Philos. Trans. Roy. Soc. B: Biol. Sci. 364 (1526), 1985. https://doi.org/10.1098/rstb.2008.0205.

Berger, P.D., 2018. Experimental Design: With Applications in Management Engineering and the Sciences. SpringerLink: Bücher, Springer, Cham. https:// doi.org/10.1007/978-3-319-64583-4.

Browne, M.A., 2015. Sources and pathways of microplastics to habitats. In: Bergmann, M., Gutow, L., Klages, M. (Eds.), Marine Anthropogenic Litter. Springer International Publishing, Cham, pp. 229-244.

Chin, C.-J.M., Fan, Z.-G., 2010. Magnetic seeding aggregation of high turbid source water. J. Environ. Eng. Manage. 20 (3), 145-150.

Chin, C.-J., Yiacoumi, S., Tsouris, C., 1998. Shear-induced flocculation of colloidal particles in stirred tanks. J. Colloid Interface Sci. 206 (2), 532-545. https://doi. org/10.1006/jcis.1998.5737.

Chin, C.-J.M., Chen, P.-W., Wang, L.-J., 2006. Removal of nanoparticles from $\mathrm{cmp}$ wastewater by magnetic seeding aggregation. Chemosphere 63 (10), 18091813. https://doi.org/10.1016/j.chemosphere.2005.09.035.

Cole, M., Lindeque, P., Halsband, C., Galloway, T.S., 2011. Microplastics as contaminants in the marine environment: a review. Marine Pollut. Bull. 62 (12), 2588-2597. https://doi.org/10.1016/j.marpolbul.2011.09.025.

Elimelech, M., 1998. Particle Deposition and Aggregation: Measurement, Modelling and Simulation, first ed., Colloid and Surface Engineering Series. ButterworthHeinemann, Woburn, Mass. https://doi.org/10.1016/B978-0-7506-7024-1. X5000-6.

Fang, M., Mishima, F., Akiyama, Y., Nishijima, S., 2010. Fundamental study on magnetic separation of organic dyes in wastewater. Phys. C: Supercond. Its Appl. 470 (20), 1827-1830. https://doi.org/10.1016/j.physc.2010.05.216.

Galgani, F., Hanke, G., Maes, T., 2015. Global distribution, composition and abundance of marine litter. In: Bergmann, M., Gutow, L., Klages, M. (Eds.), 
Marine Anthropogenic Litter. Springer International Publishing, Cham, pp. 2956.

Gouin, T., Roche, N., Lohmann, R., Hodges, G., 2011. A thermodynamic approach for assessing the environmental exposure of chemicals absorbed to microplastic Environ. Sci. Technol. 45 (4), 1466-1472. https://doi.org/10.1021/es1032025.

Jambeck, J.R., Geyer, R., Wilcox, C., Siegler, T.R., Perryman, M., Andrady, A., Narayan, R., Law, K.L., 2015. Plastic waste inputs from land into the ocean. Science 347 (6223), 768. https://doi.org/10.1126/science.1260352.

Karapinar, N., 2003. Magnetic separation of ferrihydrite from wastewater by magnetic seeding and high-gradient magnetic separation. Int. J. Miner. Process. 71 (1), 45-54. https://doi.org/10.1016/S0301-7516(03)00029-2.

Kirby, B.J., Hasselbrink Jr., E.F., 2004. Zeta potential of microfluidic substrates: 2. Data for polymers. Electrophoresis 25 (2), 203-213. https://doi.org/10.1002/ elps.200305755

Klaine, S.J., Koelmans, A.A., Horne, N., Carley, S., Handy, R.D., Kapustka, L., Nowack, B., von der Kammer, F., 2012. Paradigms to assess the environmental impact of manufactured nanomaterials. Environ. Toxicol. Chem. 31 (1), 3-14. https://doi. org/10.1002/etc.733.

Kühn, S., Bravo Rebolledo, E.L., van Franeker, J.A., 2015. Deleterious Effects of Litter on Marine Life. Springer International Publishing, Cham, pp. 75-116.

S. Ladislav, Solid-liquid separation, fourth ed., Butterworth-Heinemann, Oxford [u. a.], 2000. https://doi.org/10.1016/B978-0-7506-4568-3.X5023-8.

Lithner, D., Larsson, k., Dave, G., 2011. Environmental and health hazard ranking and assessment of plastic polymers based on chemical composition. Sci. Total Environ. 409 (18), 3309-3324. https://doi.org/10.1016/j.scitotenv.2011.04.038.

Luce, H.R., 1955. Throwaway living: dozens of disposable housewares eliminate the chores of cleaning up. LIFE 39 (5), 43-44.

Menzel, K., Windt, C.W., Lindner, J.A., Michel, A., Nirschl, H., 2013. Dipolar openable Halbach magnet design for high-gradient magnetic filtration. Sep. Purif. Technol. 105 (Supplement C), 114-120. https://doi.org/10.1016/j. seppur.2012.12.019.

PlasticsEurope, Plastics - the facts 2018, Report (2018). URL plasticseurope.org.

Rhein, F., Ruß, F., Nirschl, H., 2019. Collision case model for population balance equations in agglomerating heterogeneous colloidal systems: Theory and experiment. Colloids Surf. A: Physicochem. Eng. Aspects 572, 67-78. https:// doi.org/10.1016/j.colsurfa.2019.03.089.

Rochman, C.M., 2015. The Complex Mixture, Fate and Toxicity of Chemicals Associated with Plastic Debris in the Marine Environment. Springer International Publishing, Cham, pp. 117-140.

Rochman, C.M., Browne, M.A., Halpern, B.S., Hentschel, B.T., Hoh, E., Karapanagioti, H.K., Rios-Mendoza, L.M., Takada, H., Teh, S., Thompson, R.C., 2013. Classify plastic waste as hazardous. Nature 494, 169. https://doi.org/10.1038/494169a.

Spelter, LE. Steiwand, A. Nirschl, H. 2010. Processing of dispersions containing fine particles or biological products in tubular bowl centrifuges. Chem. Eng. Sci. 65 (14), 4173-4181. https://doi.org/10.1016/j.ces.2010.04.028.

Sun, Z.-X., Su, F.-W., Forsling, W., Samskog, P.-O., 1998. Surface characteristics of magnetite in aqueous suspension. J. Colloid Interface Sci. 197 (1), 151-159. https://doi.org/10.1006/jcis.1997.5239.

Svoboda, J., 2004. Magnetic Techniques for the Treatment of Materials. Springer, Netherlands. https://doi.org/10.1007/1-4020-2107-0.

Taboada-Serrano, P., Chin, C.-J., Yiacoumi, S., Tsouris, C., 2005. Modeling aggregation of colloidal particles. Curr. Opin. Colloid Interface Sci. 10 (3), 123-132. https:// doi.org/10.1016/j.cocis.2005.07.003

Terashima, Y., Ozaki, H., Sekine, M., 1986. Removal of dissolved heavy metals by chemical coagulation, magnetic seeding and high gradient magnetic filtration. Water Res. 20 (5), 537-545. https://doi.org/10.1016/0043-1354(86)90017-5.

Tsouris, C., Yiacoumi, S., Scott, T.C., 1995. Kinetics of heterogeneous magnetic flocculation using a bivariate population-balance equation. Chem. Eng. Commun. 137 (1), 147-159. https://doi.org/10.1080/00986449508936373.

Wan, T.-J., Shen, S.-M., Siao, S.-H., Huang, C.-F., Cheng, C.-Y., 2011. Using magnetic seeds to improve the agregation and precipitation of nanoparticles from backside grinding wastewater. Water Res. 45 (19), 6301-6307. https://doi.org/ 10.1016/j.watres.2011.08.067.

Wu, N., Wyart, Y., Liu, Y., Rose, J., Moulin, P., 2013. An overview of solid/liquid separation methods and size fractionation techniques for engineered nanomaterials in aquatic environment. Environ. Technol. Rev. 2 (1), 55-70. https://doi.org/10.1080/09593330.2013.788073. 
Karlsruher Institut für Technologie

\section{Repository KITopen}

Dies ist ein Postprint/begutachtetes Manuskript.

Empfohlene Zitierung:

Rhein, F.; Scholl, F.; Nirschl, H.

Magnetic seeded filtration for the separation of fine polymer particles from dilute suspensions: Microplastics. 2019. Chemical engineering science, 207.

doi: $\underline{10.5445 / / R / 1000097236}$

Zitierung der Originalveröffentlichung:

Rhein, F.; Scholl, F.; Nirschl, H.

Magnetic seeded filtration for the separation of fine polymer particles from dilute suspensions: Microplastics.

2019. Chemical engineering science, 207, 1278-1287.

doi:10.1016/j.ces.2019.07.052

Lizenzinformationen: CC BY-NC-ND 4.0 